\title{
Image-Based Machine Learning Algorithms for Disease Characterization in the Human Type 1 Diabetes Pancreas
}

Xiaohan Tang, ${ }^{* \dagger}$ Irina Kusmartseva, ${ }^{*}$ Shweta Kulkarni, ${ }^{*}$ Amanda Posgai, ${ }^{*}$ Stephan Speier, ${ }^{\ddagger 5}$ Desmond A. Schatz, Michael J. Haller, Martha Campbell-Thompson, ${ }^{* * *}{ }^{*}$ Clive H. Wasserfall, ${ }^{*}$ Bart 0. Roep ${ }^{\dagger \dagger}$ John S. Kaddis, ${ }^{\dagger \dagger * *}$ and Mark A. Atkinson* ${ }^{*}$

From the Departments of Pathology, Immunology and Laboratory Medicine* and Pediatrics, $"$ College of Medicine, University of Florida Diabetes Institute, Gainesville, Florida; the Department of Metabolism and Endocrinology, ${ }^{\dagger}$ The Second Xiangya Hospital, Central South University, Changsha, China; the Paul Langerhans Institute Dresden of the Helmholtz Zentrum München at the University Clinic Carl Gustav Carus of Technische Universität Dresden, ${ }^{\ddagger}$ Helmholtz Zentrum München, Neuherberg, Germany; the Institute of Physiology, ${ }^{\S}$ Faculty of Medicine, Technische Universität Dresden, Dresden, Germany; the German Center for Diabetes Research, "München-Neuherberg, Germany; the J. Crayton Pruitt Family Department of Biomedical Engineering, ** University of Florida, Gainesville, Florida; and the Departments of Diabetes Immunology ${ }^{\dagger \dagger}$ and Diabetes and Cancer Discovery Science, ${ }^{\ddagger \ddagger}$ Diabetes and Metabolism Research Institute, City of Hope/Beckman Research Institute, Duarte, California

Accepted for publication November 24, 2020.

Address correspondence to Mark A. Atkinson, Ph.D., Department of Pathology, University of Florida, College of Medicine, Box 100275, 1275 Center Dr., BMSB J593, Gainesville, FL 32610. E-mail: atkinson@ufl.edu.

\begin{abstract}
Emerging data suggest that type 1 diabetes affects not only the $\beta$-cell-containing islets of Langerhans, but also the surrounding exocrine compartment. Using digital pathology, machine learning algorithms were applied to high-resolution, whole-slide images of human pancreata to determine whether the tissue composition in individuals with or at risk for type 1 diabetes differs from those without diabetes. Transplantgrade pancreata from organ donors were evaluated from 16 nondiabetic autoantibody-negative controls, 8 nondiabetic autoantibody-positive subjects with increased type 1 diabetes risk, and 19 persons with type 1 diabetes ( 0 to 12 years' duration). HALO image analysis algorithms were implemented to compare architecture of the main pancreatic duct as well as cell size, density, and area of acinar, endocrine, ductal, and other nonendocrine, nonexocrine tissues. Type 1 diabetes was found to affect exocrine area, acinar cell density, and size, whereas the type of difference correlated with the presence or absence of insulin-positive cells remaining in the pancreas. These changes were not observed before disease onset, as indicated by modeling cross-sectional data from pancreata of autoantibody-positive subjects and those diagnosed with type 1 diabetes. These data provide novel insights into anatomic differences in type 1 diabetes pancreata and demonstrate that machine learning can be adapted for the evaluation of disease processes from crosssectional data sets. (Am J Pathol 2021, 191: 454-462; https://doi.org/10.1016/j.ajpath.2020.11.010)
\end{abstract}

Once thought a disease primarily affecting insulin-producing $\beta$ cells, emerging evidence suggests that type 1 diabetes affects both the endocrine and exocrine pancreatic compartments. The exocrine pancreas, which comprises $\geq 80 \%$ of the organ's mass, is responsible for the synthesis and secretion of digestive enzymes that flow via a series of converging ducts to the main pancreatic duct, and ultimately to the duodenum, where they aid in food digestion. ${ }^{1,2}$ Recent studies highlighting the potential role

\footnotetext{
Supported by the Network for Pancreatic Organ Donors with Diabetes (nPOD) RRID:SCR_014541; a collaborative type 1 diabetes research project sponsored by the Juvenile Diabetes Research Foundation nPOD:5SRA-2018-557-Q-R， 25-2013-268， 25-2012-380, and 25-2007-874 (M.A.A.), including a subcontract (J.S.K.); The Leona M. \& Harry B. Helmsley Charitable Trust grant 2018PG-T1D053; the NIH National Institute of Diabetes and Digestive and Kidney Diseases-supported Human Islet Research Network RRID:SCR_014393 U24 DK104162 (J.S.K.); NIH
}

National Institute of Allergy and Infectious Diseases program project grant P01 AI042288 (M.A.A.); NIH Common Fund supported Stimulating Peripheral Activity to Relieve Conditions program grant OT2 OD023861 (M.C.-T.); and NIH National Institute of Diabetes and Digestive and Kidney Diseases grants R01 DK122160 (M.C.T.), UC4 DK108132 (M.A.A.), and R01 DK124292 (M.A.A. and S.S.).

Disclosures: None declared. 
of the exocrine pancreas in type 1 diabetes have found the following: i) pancreata are up to $50 \%$ smaller (as measured by weight or volume) in persons with type 1 diabetes, approximately $25 \%$ smaller in nondiabetic islet autoantibody positive $\left(\mathrm{AAb}^{+}\right)$individuals, and about $10 \%$ smaller in autoantibody negative $\left(\mathrm{AAb}^{-}\right)$first-degree relatives of type 1 diabetes patients who share a genetic burden for the disease, compared with nondiabetic $\mathrm{AAb}^{-}$controls $^{3-7}$; ii) lower serum trypsinogen levels in $\mathrm{AAb}^{+}$subjects who have stage 1 to 2 preclinical type 1 diabetes $^{8}$; iii) higher complement deposition throughout the vessels and ducts of the exocrine region in type 1 diabetes pancreata $^{9}$; iv) lower exocrine enzyme levels, assessed from stool samples from patients with type 1 diabetes ${ }^{10}$; v) higher immune cell infiltration within the acinar region of the exocrine pancreas from persons with type 1 diabetes $^{11}$; and vi) loss of peri-islet amylase-negative cell clusters in type 1 diabetes pancreata. ${ }^{12}$ Given that the exocrine pancreas constitutes the largest portion of cell mass, we hypothesized that the aforementioned findings on pancreatic weight/volume might be attributable to changes or abnormalities in the main pancreatic duct or that fewer or smaller acinar cells would exist in the pancreas from those with or at increased risk for type 1 diabetes. Indeed, Wright et al ${ }^{13}$ recently reported that acinar cells within pancreata from persons with long-duration type 1 diabetes were fewer in number but comparable in size to those of age-matched controls. However, more importantly, a quantitative description of exocrine and ductal pancreas tissues before and after type 1 diabetes onset is lacking.

Digital pathology is a rapidly evolving area of clinical and investigative pathology. For many diseases, important clues to pathogenesis are embedded in microscopic tissue sections. However, a reoccurring issue in the assessment of pathology specimens is related to its subjective nature, leading to high interobserver variability. ${ }^{14}$ Moreover, subtle changes in measurable parameters that can potentially enhance tissue characterization are not always apparent or are difficult to accurately quantify for human visual inspections. ${ }^{15}$ Recent advances in a computer-aided examination of tissue sections offer accuracy, efficiency, and reproducibility of extracted information. ${ }^{16,17}$ The development of machine and deep learning algorithms has enabled the characterization of changes in tissue microenvironment that could play a critical role in disease pathogenesis. ${ }^{18}$ Hence, in this study, the stateof-the-art HALO quantitative image analysis platform version 2.1 (Indica Lab, Albuquerque, NM) with built-in machine learning algorithms was used to quantify the proportion of endocrine, acinar, and ductal/other (nonendocrine, nonexocrine tissues) tissue areas, as well as acinar and endocrine cell density and size, in subjects with or at risk for type 1 diabetes, as well as in controls without type 1 diabetes.

\section{Materials and Methods}

\section{Donors and Samples}

Pancreata from cadaveric organ donors were recovered according to federal guidelines and as approved by the University of Florida Institutional Review Board. Pancreata were processed by the Network for Pancreatic Organ Donors with Diabetes, as previously described. ${ }^{19,20}$ Organ Procurement Organizations partnering with the Network for Pancreatic Organ Donors with Diabetes to provide research resources are listed (http://www.jdrfnpod.org//for-partners/npod-partners, last accessed November 12, 2020). A total of 16 nondiabetic $\mathrm{AAb}^{-}$persons, 8 nondiabetic $\mathrm{AAb}^{+}$persons with increased type 1 diabetes risk, and 19 type 1 diabetes donors were evaluated, and statistical testing to verify matching was performed (Supplemental Tables S1, S2, and S3). Herein, increased risk is defined as the presence of $\geq 1$ islet AAb in the absence of the disease, which is considered pre-stage 1 to stage 2 preclinical type 1 diabetes. ${ }^{21}$ An extensive body of literature supports the notion that fewer single $\mathrm{AAb}^{+}$individuals will develop the disease than those with $\geq 2$ AAbs, although the level of risk is largely determined by number and type of AAbs. ${ }^{22-24}$

\section{Histology and Immunohistochemistry}

Each pancreas was divided into three regions: pancreatic head $(\mathrm{PH})$, pancreatic body $(\mathrm{PB})$, and pancreatic tail $(\mathrm{PT})$; each region was sectioned into sagittal blocks, followed by processing. ${ }^{20}$ Paraffin sections ( $4 \mu \mathrm{m}$ thick) from one representative tissue block within each region were stained with hematoxylin and eosin or by immunohistochemistry ${ }^{12}$ using the following primary antibodies: rabbit monoclonal anti-insulin (RRID: AB_2716761; 1:2000 dilution; Abcam, Cambridge, MA) and mouse monoclonal anti-glucagon (RRID: AB_297642; 1:1000 dilution; Abcam). Stained tissue sections were digitized at $\times 20$ magnification using an Aperio CS2 slide scanner (Leica Biosystems, Inc., Wetzlar, Germany) (Figure 1A).

\section{Morphometric Analysis of Pancreatic Tissue Composition}

The digitized tissue sections were analyzed using the HALO version 2.1 quantitative image analysis platform. The annotation pen tool was used to outline the tissue section to determine total tissue area $\left(\mathrm{mm}^{2}\right)$. Using color, texture, and contextual features, the machine learning random forest tissue classifier algorithm was trained to recognize and segregate the following tissue types: i) endocrine, all cells staining positive for insulin or glucagon; ii) acinar, insulin-negative, glucagon-negative tissue, excluding regions classified as ductal/other; and iii) ductal/other, morphologically identifiable ducts, vessels, nerves, fibrotic tissue, and adipose tissue (Figure 1B). The tissue classifier was then applied to calculate areas of all tissue types in each pancreatic region. The proportional area of each tissue type (percentage) was calculated as the tissue type area divided by total tissue area. The Cytonuclear Algorithm (Indica Lab) version 1.6 was then used for calculating average tissue type cell numbers and size for each donor and pancreas region. The algorithm threshold parameters were set to detect all nuclei and to 
discriminate endocrine cells on the basis of their immunohistochemistry immunopositivity. The algorithm was applied across all images, and each image was automatically quantified (Figure 1C). Total cell number and average cell area (size) were reported for both endocrine and acinar tissue. Cell size was ranked into 20 groups, which were binned into three categories for visualization purposes only. Endocrine and acinar cell densities were calculated as cell count in tissue type/total class area measured.

\section{Main Pancreatic Duct Quantification}

The HALO annotation pen tool was used to outline the main pancreatic duct on hematoxylin and eosin images for internal wall (bounded by the ductal epithelium) and external wall (formed by bordering acinar cells). Total area was measured as the area within the external wall, including luminal space. The thickness was estimated as the distances between internal and external walls. Measurements $(n=9$ to 19 per duct) were collected using a ruler tool at evenly spaced intervals around the entire duct lumen (Figure 2A).

\section{Statistical Analysis}

Pancreas weights, defined as a percentage of whole-body weight (termed relative pancreatic weight), were calculated as previously described. ${ }^{5}$ Because pancreas weight has been shown to mature during early postnatal stages in life, ${ }^{25}$ but generally plateau by the age of 20 years ${ }^{26}$ the analysis of pancreas weight was stratified by age as follows: $\leq 1$ year of age was classified as developmental or neonatal, $>1$ and $\leq 12$ years of age was classified as childhood, $>12$ to $\leq 18$ years of age was classified as adolescence, and $>18$ years of age was classified as adulthood. This grouping is consistent with previously described quantified $\beta$-cell formation in stages throughout life. ${ }^{27}$ Generalized linear mixed effect models were used to evaluate differences in relative pancreatic weight by donor group or length of diabetes duration (subgroup analysis). ${ }^{28}$ The Kenward-Roger method was used to compute denominator degrees of freedom in $t$ and $f$ tests, ${ }^{29}$ and different intercepts were fit for each individual to address subject-to-subject variation.

For generalized linear mixed effect models involving independent variables related to endocrine, acinar, ductal, and main duct end points, differences by pancreas region and donor group, and the interaction of the two were evaluated. Pancreas region was treated as a repeated measurement variable, and different intercepts were fit for each individual. Homogeneous and heterogeneous covariance structures (eight total) were examined for each model generated; final selection was based on the use of Akaike and Bayesian Information Criteria.

For all model-based data, point estimates and $95 \%$ CIs of least square mean differences between groups or pancreas region were obtained; adjustments to $P$ values and CIs for individual comparisons were made using the method of
Tukey-Kramer. Interactions were reported if found to be significant, and simple main effects included. All $P$ values are two sided and statistically significant if $P<0.05$, unless otherwise noted. Statistical analyses were performed using SAS (SAS Insitute Inc., Cary, NC) version 9.4 TS Level 1M1. Jupyter Notebooks were prepared to enable sharing of data and reproduction of all results and figures, available on GitHub through Zenodo (https://doi.org/10.5281/zenodo. 4269672, last accessed November 12, 2020).

\section{Results}

\section{Pancreatic Duct Anatomy in Type 1 Diabetes}

The main pancreatic duct was assessed in the $\mathrm{PH}, \mathrm{PB}$, and $\mathrm{PT}$ regions in nondiabetic $\mathrm{AAb}^{-}$controls and type 1 diabetes donors, with or without insulin-containing islets (insulinpositive and insulin-negative, respectively) (Figure 2A). No statistically significant differences were observed in the wall thickness or area of the main pancreatic duct by disease status (overall $P=0.8109$ and $P=0.6625$, respectively). However, differences in both wall thickness (overall $P<0.0001$ ) (Figure 2B) and area (overall $P<0.0001$ ) (Figure 2C) were observed by pancreas region. Specifically, the duct area was smaller $(\Delta=-0.4772 ; 95 \% \mathrm{CI},-0.7357$ to -0.2188 ; $P=0.0002)$ and thinner $(\Delta=-54.5732 ; 95 \% \mathrm{CI}$, -82.8027 to $-26.3436 ; P=0.0001)$ in the PT versus that in PB. No interactions between disease status and pancreas region were noted in the area $(P=0.9725)$ or wall thickness $(P=0.8143)$.

\section{Compositional Analysis of Type 1 Diabetes and Nondiabetic Pancreata}

Using a tissue classifier, the proportions of endocrine, acinar, and ductal/other (vessels, nerves, adipose tissue, and fibrotic tissue in the pancreas) tissue areas were measured in the $\mathrm{PH}$, PB, and PT regions (Figure 1B). Differences by disease status were observed in endocrine (overall $P<0.0001$ ), acinar (overall $P=0.0248)$, and ductal/other $(P=0.0037)$ tissue area (Figure 3, A and C). Endocrine area was smaller in type 1 diabetes subjects with residual insulin-positive islets (average disease duration, $2.3 \pm 2.6$ years) versus either nondiabetic $\mathrm{AAb}^{-}(\Delta=-1.4082 ; 95 \% \mathrm{CI},-1.9761$ to -0.8403 ; $P<0.0001)$ or $\mathrm{AAb}^{+}$donors $(\Delta=-0.8883 ; 95 \% \mathrm{CI}$, -1.5398 to $-0.2368 ; P=0.0042$ ). It was also smaller in type 1 diabetes insulin-negative versus either nondiabetic $\mathrm{AAb}^{-}$ $(\Delta=-1.4396 ; 95 \% \mathrm{CI},-2.0743$ to $-0.8048 ; P<0.0001)$ or $\mathrm{AAb}^{+}$donors $(\Delta=-0.9197 ; 95 \% \mathrm{CI},-1.6302$ to -0.2091 ; $P=0.0069$ ). Acinar area was smaller in type 1 diabetes insulin-negative versus either nondiabetic $\mathrm{AAb}^{-}$ $(\Delta=-5.9737 ; 95 \% \mathrm{CI},-11.2450$ to $-0.7023 ; P=0.0212)$ or $\mathrm{AAb}^{+}$donors $(\Delta=-5.9227 ; 95 \% \mathrm{CI},-11.8313$ to $-0.01401 ; P=0.0493)$. Ductal/other tissue area was larger in type 1 diabetes insulin-negative versus either nondiabetic $\mathrm{AAb}^{-}(\Delta=7.4160 ; 95 \% \mathrm{CI}, 2.1978-11.3440 ; P=0.0027)$ 


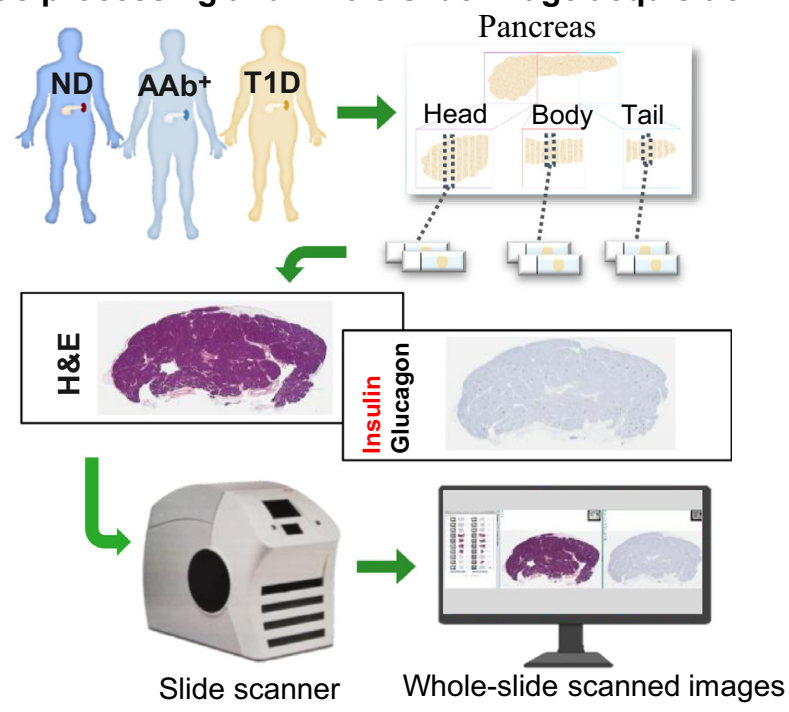

\section{B Input parameters for the tissue classifier and cytonuclear algorithms}

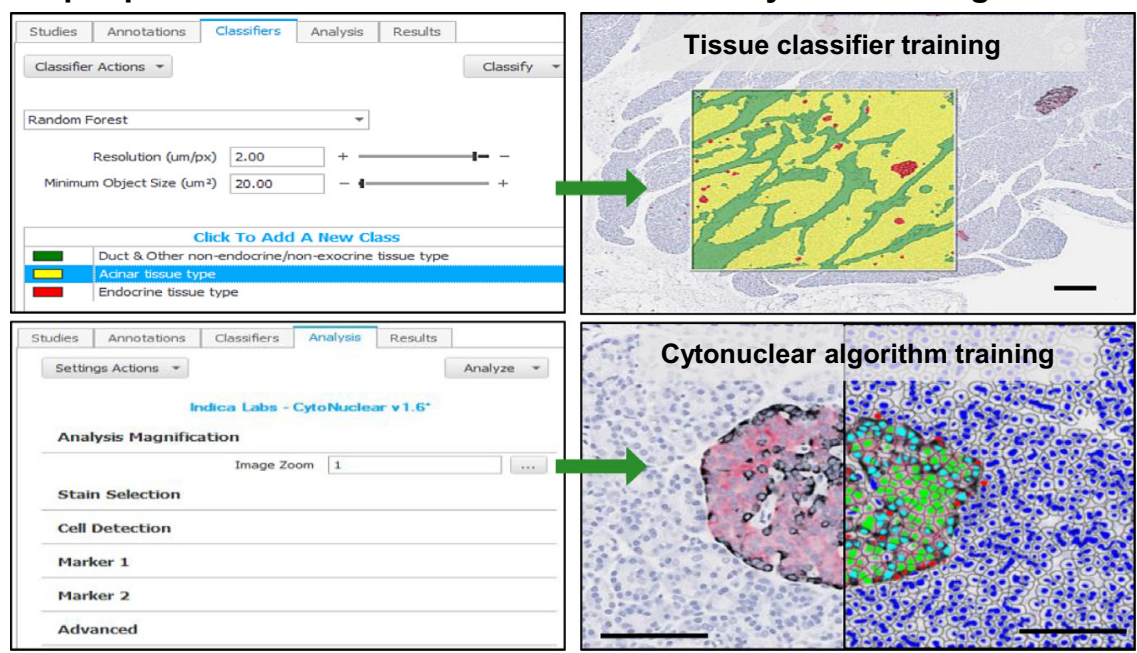

C Output data information extracted from whole tissue section

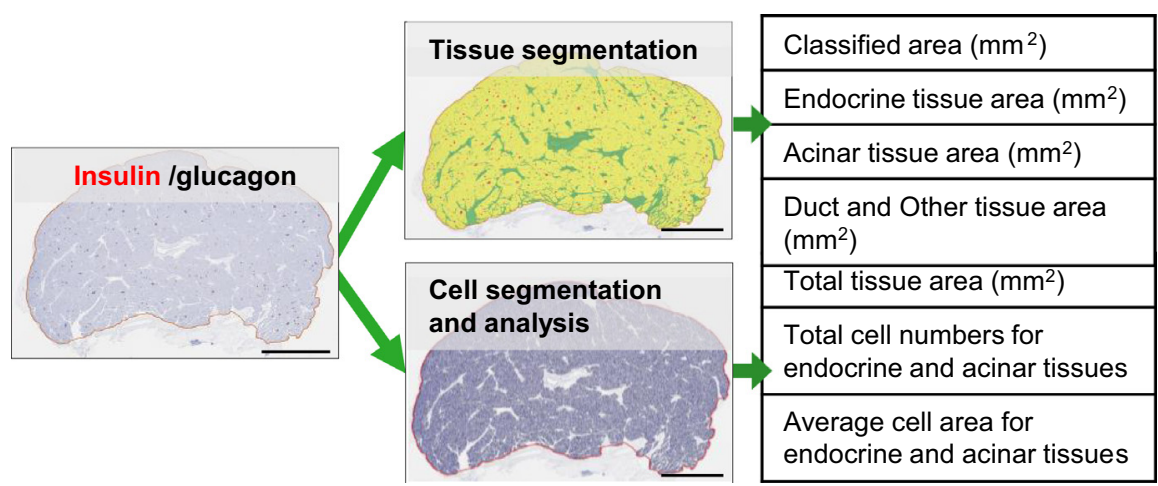

Figure 1 Tissue segmentation and cell analysis using two machine learning algorithms. A: Each pancreas from no diabetes autoantibody negative (ND); no diabetes autoantibody positive (AAb ${ }^{+}$), and type 1 diabetes (T1D) organ donors was sectioned into sagittal blocks, followed by processing into formalin-fixed, paraffin-embedded tissue blocks. Paraffin sections from one representative tissue block within each pancreatic region were stained with hematoxylin and eosin or by immunohistochemistry and digitized by the slide scanner to obtain whole-slide images. B: Three types of annotation classes were generated for training the tissue classifier algorithm to recognize and segment pancreatic tissue into three tissue types: endocrine (red), acinar (yellow), and duct and other nonendocrine/nonexocrine (green). The cytonuclear algorithm parameters were set to detect all nuclei on the image and discriminated endocrine cells on the basis of immunohistochemistry positivity. C: Once trained, both algorithms were then applied in a two-step manner across all images, and each image was automatically quantified. Scale bars: $50 \mu \mathrm{m}$ (B); $5 \mathrm{~mm}$ (C). 

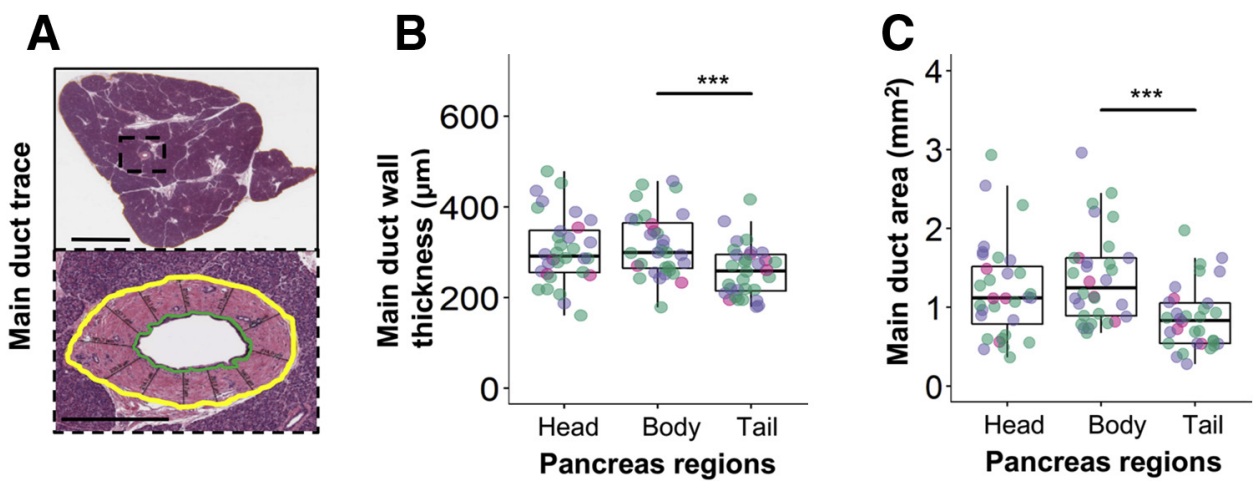

Figure 2 No observed effects of type 1 diabetes on the main pancreatic duct anatomy. A: The main pancreatic duct was identified (top panel) throughout the organ in the head, body, and tail, and measurements were taken (bottom panel). Boxed area is shown at higher magnification below. B and C: Green denotes luminal wall trace, yellow indicates duct perimeter wall, and lines between them indicate duct thickness (B). The duct area is defined as luminal space plus duct wall $(\mathbf{C})$. Green indicates those without type 1 diabetes who were autoantibody negative (nondiabetic AAb ${ }^{-}$); purple, those with type 1 diabetes with insulin-positive islets remaining in the pancreas (T1D INS ${ }^{+}$); and pink, those with type 1 diabetes without insulin-positive islets remaining in the pancreas (T1D INS ${ }^{-}$). See Results for statistically significant differences between disease groups. $n=16$ for nondiabetic AAb ${ }^{-}(\mathbf{B}$ and $\mathbf{C}) ; n=12$ for T1D INS ${ }^{+}(\mathbf{B}$ and C); $n=4$ for T1D INS ${ }^{-}$(B and C). ${ }^{* * * P}<0.001$. Scale bars: $5 \mathrm{~mm}$ (A, top panel); $500 \mu \mathrm{m}$ (A, bottom panel).

or $\mathrm{AAb}^{+}$donors $(\Delta=6.8413 ; 95 \% \mathrm{CI}, 0.9903-12.6924$; $P=0.0166$ ) (Figure 3, A and B).

Finally, the relative weights of endocrine, exocrine, and ductal/other tissues were calculated for each organ as whole pancreas weight multiplied by the ratio of each tissue type to total tissue area, and divided by the whole-body weight of that individual. The relative weights of pancreatic endocrine and acinar tissues differed across donor groups (overall $P<0.0001$ and $P=0.0308$, respectively), but not of ductal $/$ other tissue (overall $P=0.2170$ ) (Figure 3B). Multiple comparisons test revealed that the percentage of endocrine tissue was smaller in type 1 diabetes insulin-negative versus nondiabetic $\mathrm{AAb}^{-}(\Delta=-0.0016 ; 95 \% \mathrm{CI},-0.0022$ to $-0.0001 ; P<0.0001)$ or $\mathrm{AAb}^{+}$donors $(\Delta=-0.0011 ; 95 \%$ $\mathrm{CI},-0.0018$ to $-0.0004 ; P=0.0009$ ), and in type 1 diabetes insulin-positive versus nondiabetic $\mathrm{AAb}^{-}$ $(\Delta=-0.0016 ; 95 \%$ CI, -0.0022 to $-0.0010 ; P<0.0001)$ or $\mathrm{AAb}^{+}$individuals $(\Delta=-0.0011 ; 95 \% \mathrm{CI},-0.0018$ to $-0.0005 ; P=0.0004)$. No other individual comparisons were significant (after multiple comparison correction) for the acinar or ductal/other tissue types across donor groups.

Differences between regions of the pancreas were observed in endocrine (overall $P<0.0001$ ) and ductal/other tissue area (overall $P=0.0113$ ), regardless of donor group (Supplemental Figure S1). Endocrine area was greater in the PT versus either PB $(\Delta=0.7087$; $95 \%$ CI, 0.4751-0.9424; $P<0.0001)$ or $\mathrm{PH}$ region $(\Delta=0.8687 ; 95 \% \mathrm{CI}$, $0.6343-1.1030 ; P<0.0001)$. Ductal/other area was smaller in the PT versus PH $(\Delta=-3.0559 ; 95 \% \mathrm{CI},-5.4131$ to $-0.6987 ; P=0.0086)$ region.

\section{Morphologic Changes in the Pancreata of Donors with Type 1 Diabetes}

For tissues with round-like cell morphology (ie, endocrine and acinar), cell density and size were evaluated (Figure 4). Differences in endocrine and acinar cell density by disease status (overall $P<0.0001$ and $P=0.0318$, respectively), but not pancreas region (overall $P=0.3133$ and $P=0.2222$, respectively), were observed. Endocrine tissue was denser in type 1 diabetes insulin-positive versus either nondiabetic $\mathrm{AAb}^{-}(\Delta=2004.07 ; 95 \% \mathrm{CI}, 884.93-3123.21 ; P=0.0002)$ or $\mathrm{AAb}^{+}$donors $(\Delta=1726.96$; $95 \% \mathrm{CI}, 443.72-3010.20$; $P=0.0048)$; it was also denser in type 1 diabetes insulinnegative versus nondiabetic $\mathrm{AAb}^{-}(\Delta=1721.61 ; 95 \% \mathrm{CI}$, 470.82-2972.40; $P=0.0038)$ or $\mathrm{AAb}^{+}$individuals $(\Delta=1444.50 ; 95 \%$ CI, 44.9584-2844.04; $P=0.0409)$ (Figure 4A). Acinar tissue was significantly denser in only type 1 diabetes insulin-positive versus nondiabetic $\mathrm{AAb}^{-}$donors $(\Delta=1314.55 ; 95 \%$ CI, 123.16-2505.93; $P=0.0259)$ (Figure 4B). Likewise, variability in cell size was observed in endocrine and acinar tissues. Endocrine cells were smaller in type 1 diabetes insulin-positive versus either nondiabetic $\mathrm{AAb}^{-}(\Delta=-7.7155 ; 95 \% \mathrm{CI},-12.4897$ to -2.9414 ; $P=0.0006)$ or $\mathrm{AAb}^{+}$donors $(\Delta=-6.1353 ; 95 \% \mathrm{CI}$, -11.6089 to $-0.6617 ; P=0.0230$ ); they were also smaller in type 1 diabetes insulin-negative versus nondiabetic $\mathrm{AAb}^{-}$ $(\Delta=-6.7501 ; 95 \% \mathrm{CI},-12.0868$ to $-1.4135 ; P=0.0085)$ individuals (Figure 4A). Acinar cells were smaller only in type 1 diabetes insulin-positive versus nondiabetic $\mathrm{AAb}^{-}$donors $(\Delta=-6.7935 ; 95 \% \mathrm{CI},-12.0564$ to $-1.5307 ; P=0.0071)$ (Figure 4B).

\section{Discussion}

Findings that $\mathrm{AAb}^{+}$individuals at risk for type 1 diabetes and those with new-onset or established disease have smaller relative pancreatic weight and relative pancreas volume $^{3-7}$ (Supplemental Figure S2), taken together with lower serum levels of trypsinogen, ${ }^{8}$ support the hypothesis that exocrine pancreas mass and function may be subclinically altered during the preclinical phase of type 1 diabetes pathogenesis. 
A
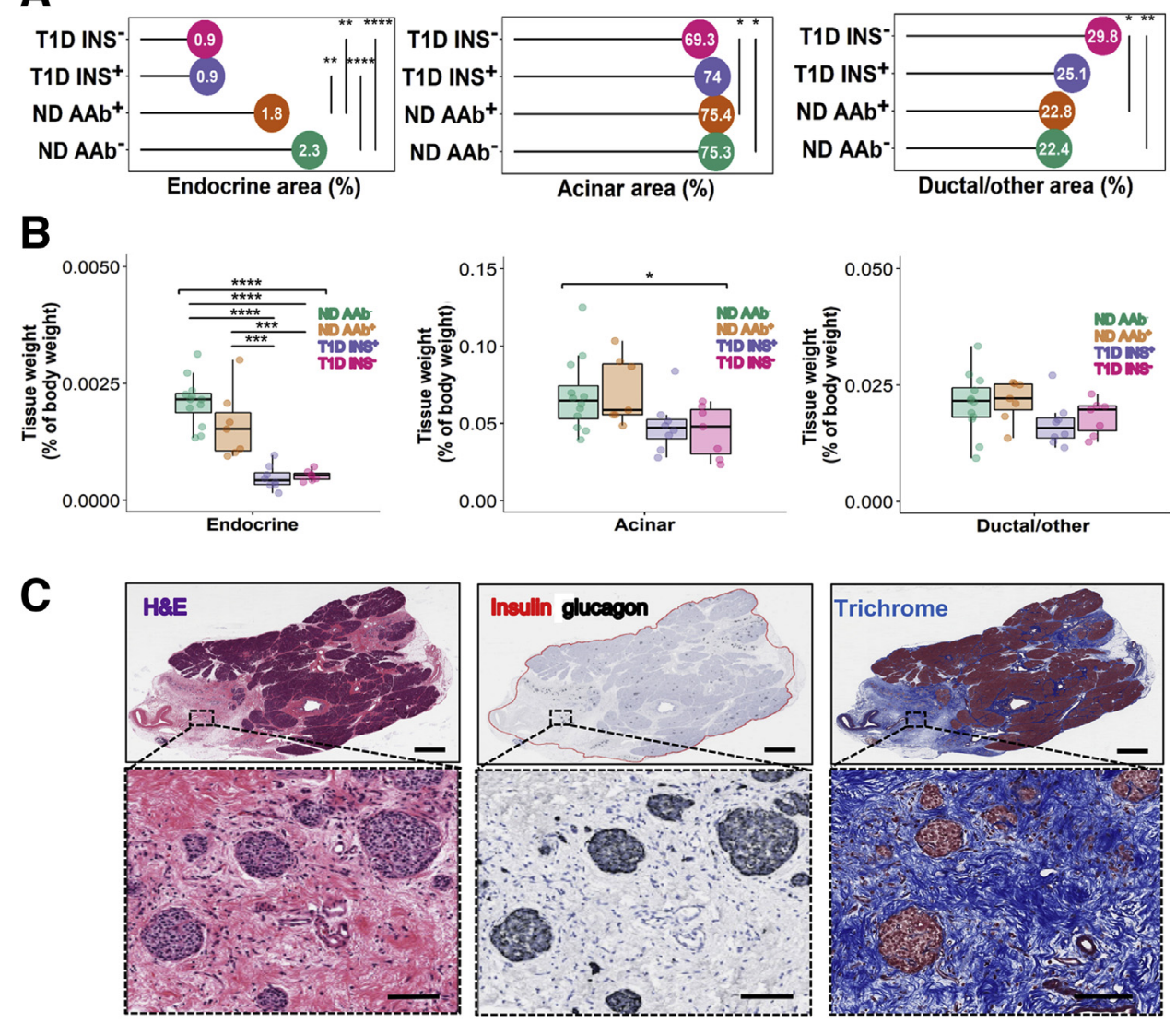

Figure 3 Compositional analysis of type 1 and nondiabetic pancreata. Tissue types were identified, then quantified as a percentage of total tissue area or body weight. A and B: For tissue type area (A) and weight (B), green represents nondiabetic autoantibody negative (ND AAb ${ }^{-}$); orange, nondiabetic autoantibody positive (ND AAb ${ }^{+}$); purple, type 1 diabetes with insulin-positive islets (T1D INS ${ }^{+}$); and pink, type 1 diabetes without insulin-positive islets (T1D INS $^{-}$). C: Images of pancreatic tissue stained for hematoxylin and eosin, insulin, glucagon, and trichrome from the tail region of Network for Pancreatic Organ Donors with Diabetes Case 6237, an 18-year-old white female with type 1 diabetes for 12 years at the time of death. Islets observed in ductal and other nonendocrine/nonexocrine tissue were devoid of contact with acinar cells. Individual differences are noted. $n=14 \mathrm{ND} \mathrm{AAb}^{-}(\mathbf{A}) ; n=12 \mathrm{ND} \mathrm{AAb}^{-}(\mathbf{B}) ; n=3$ single ND AAb $(\mathbf{A}) ; n=5$ double ND AAb ${ }^{+}(\mathbf{A}) ; n=8$ total ND AAb ${ }^{+}(\mathbf{A}) ; n=2$ single ND AAb ${ }^{+}(\mathbf{B}) ; n=5$ double ND AAb $^{+}(\mathbf{B}) ; n=7$ total ND AAb ${ }^{+}(\mathbf{B}) ;$

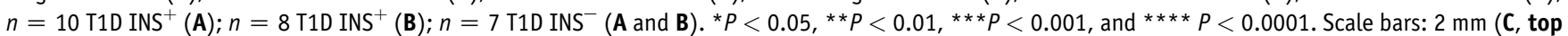
row); $100 \mu \mathrm{m}$ (C, bottom row).

Recent advances in whole-slide imaging technology, development of machine learning algorithms, and computer-based specialized image analysis software allow automated discrimination and measurements of cellular and tissue components on the digital images of tissue samples. An automated analysis of digitized tissue section generates highly reproducible quantitative tissue-derived data sets and has an advantage over manual visual quantification because of significantly reduced human bias, making it the method of choice for the evaluation of pathologic specimens. ${ }^{18}$ Thus, this study employed the state-of-the-art HALO image analysis platform with builtin machine learning algorithms to assess whether changes in pancreas mass and exocrine function in subjects with type 1 diabetes might be attributable to abnormalities affecting the main pancreatic duct and/or smaller or fewer acinar cells in pancreata from subjects with type 1 diabetes and nondiabetic $\mathrm{AAb}^{+}$individuals at increased risk of disease.
The main pancreatic duct was thinner and occupied smaller tissue area within the cross-section from the PT versus PB region, likely reflective of normal variation based on anatomic location ${ }^{30}$; however, the main pancreatic duct area in type 1 diabetes donors was comparable to nondiabetic individuals. Dilated duct has been reported in patients with insulin-dependent diabetes. ${ }^{31}$ In our study, the main pancreatic duct area was unaltered in subjects with type 1 diabetes, and the thickness of the main pancreatic duct wall was similar across all study groups.

Tissues were classified as endocrine, acinar, or duct/other (nonendocrine/nonexocrine) areas, and cell size and density of endocrine and acinar tissue within each pancreas region were calculated. Endocrine tissue area was reduced, whereas endocrine cell density was increased, in type 1 diabetes donors compared with controls, regardless of the presence of residual insulin-positive islets. Interestingly, in type 1 diabetes subjects with residual insulin-positive islets, the percentage of pancreas cross-sectional area corresponding to 
A

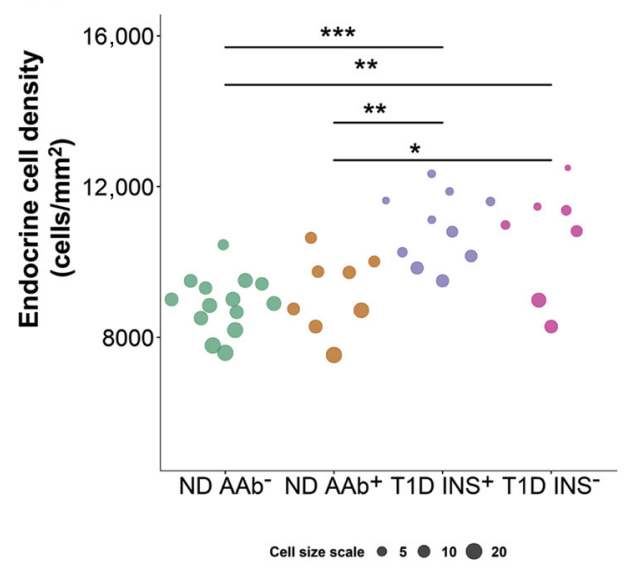

B

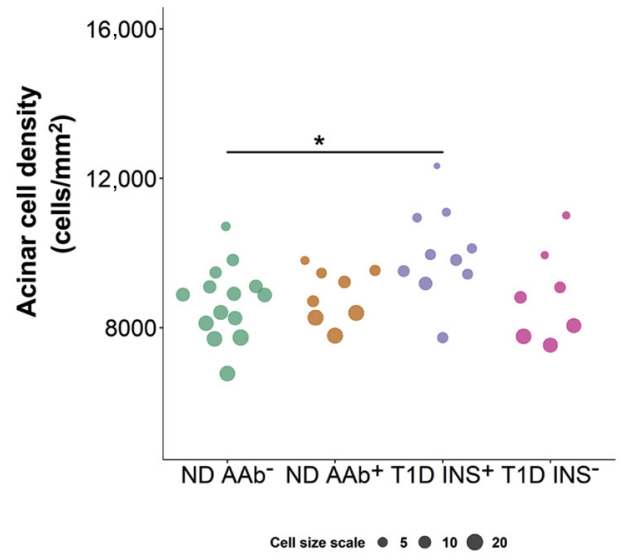

Figure 4 Morphologic changes in the pancreata of donors with type 1 diabetes. Cell size and density data were obtained from the head, body, and tail regions of each pancreas for endocrine $(\mathbf{A})$ and acinar $(\mathbf{B})$ tissue types, then averaged and plotted for each individual. Cell size was ranked and binned into three categories for visualization purposes only. Green represents nondiabetic autoantibody negative (ND AAb ${ }^{-}$; ; orange, nondiabetic autoantibody positive $\left(\mathrm{ND} \mathrm{AAb}^{+}\right)$; purple, type 1 diabetes with insulin-positive islets (T1D INS ${ }^{+}$); and pink, type 1 diabetes without insulin-positive islets (T1D INS ${ }^{-}$). See Results for additional details. Individual differences noted. $n=14 \mathrm{ND} \mathrm{AAb}^{-}(\mathbf{A}$ and $\mathbf{B}) ; n=3$ single ND AAb ${ }^{+}(\mathbf{A}$ and $\mathbf{B}) ; n=5$ double ND AAb ${ }^{+}(\mathbf{A}$ and $\mathbf{B}) ; n=8$ total

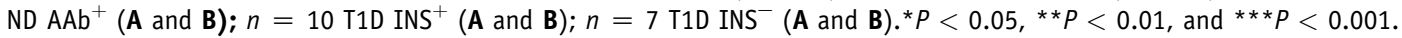

acinar tissue was similar to that in nondiabetic donors with or without AAbs, but the acinar cell density was greater. In humans, the sizes of acinar and $\beta$ cells have been shown to remain stable throughout postnatal life. ${ }^{32}$ Hence, the observed reductions in acinar cell size could reflect anatomic differences that may have existed before pathogenesis of the disease, which possibly could have contributed to type 1 diabetes development. Nevertheless, in the current study, the acinar cell size was comparable between nondiabetic $\mathrm{AAb}^{+}$donors and nondiabetic $\mathrm{AAb}^{-}$donors, suggesting that smaller acinar cells may not necessarily precede disease onset, but result from factors associated with type 1 diabetes. ${ }^{33}$ Indeed, factors such as a progressive loss of trophic effect of insulin on acinar cells due to loss of functional $\beta$-cell mass ${ }^{34}$ increased presence of proinflammatory cytokines and inflammatory cell infiltrate in exocrine pancreas, ${ }^{11}$ persistent endoplasmic reticulum stress, and altered unfolded protein response in endocrine and exocrine pancreas ${ }^{35,36}$ generate prolonged cell stress, possibly forcing cells to adapt by changing their size. ${ }^{37}$ Alternatively, different mechanisms could potentially contribute first to smaller pancreas size and reduced exocrine function before clinical diagnosis and, then, smaller acinar cell size following disease onset. In contrast, in type 1 diabetes donors without residual insulin-positive islets, the acinar cross-sectional tissue area was reduced, but that of ducts, vessels, nerves, fibrotic tissue, and adipose tissue was greater compared with that in nondiabetic $\mathrm{AAb}^{+}$or $\mathrm{AAb}^{-}$ donors. Therefore, these findings confirm earlier observations of extensive intralobular and perilobular fibrosis and severe acinar atrophy in the pancreas of patients with long-standing type 1 diabetes. ${ }^{38,39}$ The data also show that the formation of excess fibrous tissue coincides with acinar tissue loss, suggesting that complete loss of insulin accompanied by longstanding autoimmunity promote structural alterations within the exocrine compartment, leading to the changes in organ tissue composition in type 1 diabetes. Although the algorithm employed in this study cannot differentiate between acinar and immune cells, the reported findings are unlikely to be altered by the presence of immune cell infiltration in the exocrine pancreas tissue, which has been reported to similarly affect insulin-containing and insulin-negative pancreata from individuals with type 1 diabetes. ${ }^{11}$

The data reported herein provide important insights into anatomic differences within the human pancreas at various stages of type 1 diabetes and further emphasize interactions between endocrine and exocrine cells during disease pathogenesis. Although the previous observations of reduced organ mass and exocrine function before and following type 1 diabetes onset ${ }^{3-8}$ suggest that such variations could conceivably precede diagnosis, the current findings support the notion that the factors associated with type 1 diabetes pathogenesis have an influence on the exocrine compartment, and possibly stipulate the multifactorial etiology of diabetic pancreatic atrophy. Further research is needed to elucidate the events leading to whole-organ defects and their possible role in potentiating type 1 diabetes progression.

\section{Acknowledgment}

We thank the families of the organ donors for the gift of tissues.

\section{Author Contributions}

X.T. and I.K. researched the data and reviewed/edited the manuscript; A.P. wrote the manuscript; S.K., M.C.-T., S.S., D.A.S., M.J.H., C.H.W., and B.R. contributed to discussion 
and reviewed/edited the manuscript; J.K. researched the data, performed statistical analysis, and reviewed/edited manuscript; M.A.A. conceived the study and reviewed/ edited the manuscript. As guarantor of this work, M.A.A. had full access to all of the data and takes full responsibility for the integrity of the data and accuracy of analysis.

\section{Supplemental Data}

Supplemental material for this article can be found at http://doi.org/10.1016/j.ajpath.2020.11.010.

\section{References}

1. Alexandre-Heymann L, Mallone R, Boitard C, Scharfmann R, Larger E: Structure and function of the exocrine pancreas in patients with type 1 diabetes. Rev Endocr Metab Disord 2019, 20:129-149

2. Hegyi P, Petersen OH: The exocrine pancreas: the acinar-ductal tango in physiology and pathophysiology. Rev Physiol Biochem Pharmacol 2013, 165:1-30

3. Campbell-Thompson M, Wasserfall C, Montgomery EL, Atkinson MA, Kaddis JS: Pancreas organ weight in individuals with disease-associated autoantibodies at risk for type 1 diabetes. JAMA 2012, 308:2337-2339

4. Williams AJ, Thrower SL, Sequeiros IM, Ward A, Bickerton AS, Triay JM, Callaway MP, Dayan CM: Pancreatic volume is reduced in adult patients with recently diagnosed type 1 diabetes. J Clin Endocrinol Metab 2012, 97:E2109-E2113

5. Campbell-Thompson ML, Kaddis JS, Wasserfall C, Haller MJ, Pugliese A, Schatz DA, Shuster JJ, Atkinson MA: The influence of type 1 diabetes on pancreatic weight. Diabetologia 2016, 59: 217-221

6. Campbell-Thompson ML, Filipp SL, Grajo JR, Nambam B, Beegle R, Middlebrooks EH, Gurka MJ, Atkinson MA, Schatz DA, Haller MJ: Relative pancreas volume is reduced in first-degree relatives of patients with type 1 diabetes. Diabetes Care 2019, 42: 281-287

7. Virostko J, Williams J, Hilmes M, Bowman C, Wright JJ, Du L, Kang H, Russell WE, Powers AC, Moore DJ: Pancreas volume declines during the first year after diagnosis of type 1 diabetes and exhibits altered diffusion at disease onset. Diabetes Care 2019, 42: 248-257

8. Li X, Campbell-Thompson M, Wasserfall CH, McGrail K, Posgai A, Schultz AR, Brusko TM, Shuster J, Liang F, Muir A, Schatz D, Haller MJ, Atkinson MA: Serum trypsinogen levels in type 1 diabetes. Diabetes Care 2017, 40:577-582

9. Rowe P, Wasserfall C, Croker B, Campbell-Thompson M, Pugliese A, Atkinson M, Schatz D: Increased complement activation in human type 1 diabetes pancreata. Diabetes Care 2013, 36:3815-3817

10. Piciucchi M, Capurso G, Archibugi L, Delle Fave MM, Capasso M, Delle Fave G: Exocrine pancreatic insufficiency in diabetic patients: prevalence, mechanisms, and treatment. Int J Endocrinol 2015, 2015 : 595649

11. Rodriguez-Calvo T, Ekwall O, Amirian N, Zapardiel-Gonzalo J, von Herrath MG: Increased immune cell infiltration of the exocrine pancreas: a possible contribution to the pathogenesis of type 1 diabetes. Diabetes 2014, 63:3880-3890

12. Kusmartseva I, Beery M, Hiller H, Padilla M, Selman S, Posgai A, Nick HS, Campbell-Thompson M, Schatz DA, Haller MJ, Wasserfall $\mathrm{CH}$, Atkinson MA: Temporal analysis of amylase expression in control, autoantibody-positive, and type 1 diabetes pancreatic tissues. Diabetes 2020, 69:60-66
13. Wright J, Saunders D, Dai C, Poffenberger G, Cairns B, Serreze D, Harlan D, Bottino R, Brissova M, Powers A: Decreased pancreatic acinar cell number in type 1 diabetes. Diabetologia 2020, 63: $1418-1423$

14. Elmore JG, Nelson HD, Pepe MS, Longton GM, Tosteson AN, Geller B, Onega T, Carney PA, Jackson SL, Allison KH, Weaver DL: Variability in pathologists' interpretations of individual breast biopsy slides: a population perspective. Ann Intern Med 2016, 164:649-655

15. Kissler H, Niland J, Olack B, Ricordi C, Hering B, Naji A, Faoud K, Oberholzer J, Fernandez L, Contreras J, Stiller T, Sowinski J, Kaufman D: Validation of methodologies for quantifying isolated human islets: an islet cell resources study. Clin Transpl 2010, 24: 236-242

16. Litjens G, Sánchez C, Timofeeva N, Hermsen M, Nagtegaal I, Kovacs I, Hulsbergen-van de Kaa C, Bult P, van Ginneken B, van der Laak J: Deep learning as a tool for increased accuracy and efficiency of histopathological diagnosis. Sci Rep 2016, 6:26286

17. Stidham R, Liu W, Bishu S, Rice M, Higgins P, Zhu J, Nallamothu B, Waljee A: Performance of a deep learning model vs human reviewers in grading endoscopic disease severity of patients with ulcerative colitis. JAMA Network Open 2019, 2:e193963

18. Aeffner F, Zarella MD, Buchbinder N, Bui MM, Goodman MR, Hartman DJ, Lujan GM, Molani MA, Parwani AV, Lillard K, Turner OC, Vemuri VNP, Yuil-Valdes AG: Introduction to digital image analysis in whole-slide imaging: a white paper from the digital pathology association. J Pathol Inform 2019, 10:9

19. Campbell-Thompson M, Wasserfall C, Kaddis J, AlbaneseO'Neill A, Staeva T, Nierras C, Moraski J, Rowe P, Gianani R, Eisenbarth G, Crawford J, Schatz D, Pugliese A, Atkinson M: Network for Pancreatic Organ Donors with Diabetes (nPOD): developing a tissue biobank for type 1 diabetes. Diabetes Metab Res Rev 2012, 28:608-617

20. Pugliese A, Yang MD, Kusmarteva I, Heiple T, Vendrame F, Wasserfall C, Rowe P, Moraski JM, Ball S, Jebson L, Schatz DA, Gianani R, Burke GW, Nierras C, Staeva T, Kaddis JS, CampbellThompson M, Atkinson MA: The Juvenile Diabetes Research Foundation Network for Pancreatic Organ Donors with Diabetes (nPOD) Program: goals, operational model and emerging findings. Pediatr Diabetes 2014, 15:1-9

21. Insel RA, Dunne JL, Atkinson MA, Chiang JL, Dabelea D, Gottlieb PA, Greenbaum CJ, Herold KC, Krischer JP, Lernmark A, Ratner RE, Rewers MJ, Schatz DA, Skyler JS, Sosenko JM, Ziegler AG: Staging presymptomatic type 1 diabetes: a scientific statement of JDRF, the Endocrine Society, and the American Diabetes Association. Diabetes Care 2015, 38:1964-1974

22. Pihoker C, Gilliam L, Hampe C, Lernmark A: Autoantibodies in diabetes. Diabetes 2005, 54(Suppl 2):S52-S61

23. Winter W, Schatz D: Autoimmune markers in diabetes. Clin Chem 2011, 57:168-175

24. Ziegler AG, Rewers M, Simell O, Simell T, Lempainen J, Steck A, Winkler C, Ilonen J, Veijola R, Knip M, Bonifacio E, Eisenbarth GS Seroconversion to multiple islet autoantibodies and risk of progression to diabetes in children. JAMA 2013, 309:2473-2479

25. Bogin B: Evolutionary perspective on human growth. Annu Rev Anthropol 1999, 28:109-153

26. Cole TJ: Growth and organ development. Adv Exp Med Biol 2009, 639:1-13

27. Gregg BE, Moore PC, Demozay D, Hall BA, Li M, Husain A, Wright AJ, Atkinson MA, Rhodes CJ: Formation of a human betacell population within pancreatic islets is set early in life. J Clin Endocrinol Metabol 2012, 97:3197-3206

28. Kim H-Y: Statistical notes for clinical researchers: assessing normal distribution (2) using skewness and kurtosis. Restor Dent Endod 2013, 38:52-54

29. Kenward MG, Roger JH: Small sample inference for fixed effects from restricted maximum likelihood. Biometrics 1997, 53:983-997 
30. Mortelé K, Rocha T, Streeter J, Taylor A: Multimodality imaging of pancreatic and biliary congenital anomalies. Radiographics 2006, 26: 715-731

31. Nakanishi K, Kobayashi T, Miyashita H, Okubo M, Sugimoto T, Murase T, Hashimoto M, Fukuchi S, Kosaka K: Exocrine pancreatic ductograms in insulin-dependent diabetes mellitus. Am J Gastroenterol 1994, 89:762-766

32. Anzi S, Stolovich-Rain M, Klochendler A, Fridlich O, Helman A, Paz-Sonnenfeld A, Avni-Magen N, Kaufman E, Ginzberg M, Snider D, Ray S, Brecht M, Holmes M, Meir K, Avivi A, Shams I, Berkowitz A, Shapiro A, Glaser B, Ben-Sasson S, Kafri R, Dor Y: Postnatal exocrine pancreas growth by cellular hypertrophy correlates with a shorter lifespan in mammals. Dev Cell 2018, 45:726-737

33. Atkinson MA, von Herrath M, Powers AC, Clare-Salzler M: Current concepts on the pathogenesis of type 1 diabetes-considerations for attempts to prevent and reverse the disease. Diabetes Care 2015, 38: 979-988
34. Logsdon C: Stimulation of pancreatic acinar cell growth by CCK, epidermal growth factor, and insulin in vitro. Am J Physiol 1986, 251:G487-G494

35. Yatchenko Y, Horwitz A, Birk R: Endocrine and exocrine pancreas pathologies crosstalk: insulin regulates the unfolded protein response in pancreatic exocrine acinar cells. Exp Cell Res 2019, 375:28-35

36. Brozzi F, Eizirik DL: ER stress and the decline and fall of pancreatic beta cells in type 1 diabetes. Ups J Med Sci 2016, 121:133-139

37. Amodeo A, Skotheim J: Cell-size control. Cold Spring Harb Perspect Biol 2016, 8:a019083

38. Gepts W: Pathologic anatomy of the pancreas in juvenile diabetes mellitus. Diabetes 1965, 14:619-633

39. Foulis AK, Stewart JA: The pancreas in recent-onset type 1 (insulindependent) diabetes mellitus: insulin content of islets, insulitis and associated changes in the exocrine acinar tissue. Diabetologia 1984 $26: 456-461$ 